\title{
GEOSPATIAL SERVICE WEB: FROM DATA, INFORMATION, AND KNOWLEDGE SHARING TO WEB GEOINTELLIGENCE
}

\author{
Jianya Gong, Huayi Wu, Peng Yue*, Xinyan Zhu, Wenxiu Gao \\ ${ }^{\text {a }}$ LIESMARS, Wuhan University, 129 Luoyu Road, Wuhan, 430079, China \\ Commission IV, WG IV/4
}

KEY WORDS: Geospatial Service Web, MapWorld, Web GeoIntelligence, Geospatial Infrastructure, Web Service, GIS

\begin{abstract}
:
The existing Spatial Data Infrastructure applications or virtual globes allow users to experiment with these tools for sharing and exploration of geospatial data. A typical application is the recently launched MapWorld, an interactive geospatial data browser developed based on GeoGlobe. Geospatial Service Web goes further by providing a geospatial infrastructure that integrates various geospatial-related resources through the Web and service technologies. The development of the Geospatial Service Web will be the establishment of a platform where data, information, and knowledge can be shared and exchanged in an interoperable and interworkable way. The fulfilment of this platform requires the integration of geospatial data acquisition system, data transformation system, distributed spatial data collection, high-capability server system, and large volume storage system etc., and exploration and extraction of useful information and knowledge from oceans of data. These are together known as Web-based Geospatial Intelligence (i.e. Web GeoIntelligence), which is an important content of the Geospatial Service Web.
\end{abstract}

\section{INTRODUCTION}

Nowadays, the development of Internet, Web services, highperformance computing, networking, and other distributed technologies provides a networked information environment for scientific research. The Geographic Information System (GIS) is now in a new network information environment, where the user groups, application and research areas, service modes, data sources, and visualization methodologies are undergoing significant changes, including:

(1) The main user groups include both the general public users and GIS professional users, while previously GIS is intended for only GIS professional users;

(2) The application and research area has developed from original isolated ones into cross-disciplinary applications and collaborative research;

(3) The visualization is involved from simple Web image browsing into a network three-dimensional virtual Earth that can allow all-round view of and modelling of the Earth;

(4) The data to be processed is now changed from static geographic data into the dynamic object sequence with spatial and temporal properties collected using multiple sensors;

(5) Simulation and large-scale computing has became one of the major means for scientific analyzing and forecasting;

(6) Service mode has changed from the simple on-line data browsing into knowledge-based intelligent network services.

The existing Spatial Data Infrastructure applications or virtual globes allow users to experiment with these tools for sharing and exploration of geospatial data in the world map. More and more geographic information can be obtained from any access device. At the same time, people can also become geographic information providers. The networked information infrastructure that connects people and all devices can provide real-time data acquisition, massive data management, highperformance computing and visualization capabilities. Geospatial Service Web (GSW) is such a geospatial infrastructure that integrates various geospatial-related resources through the Web and service technologies (Gong et al., 2009). The development of the GSW will be the establishment of a platform where data, information, and knowledge can be shared and exchanged in an interoperable and interworkable way. How to integrate different data sharing and processing functions, mine useful information from structured and unstructured, dynamic, and complex data, develop a series of related methods, techniques, and middleware platforms for solution of scientific application problems (such as resources environment, public health, urban planning, disaster prevention, emergency response) become the major tasks of the Web-based Geospatial Intelligence, shortly known as Web GeoIntelligence, which is an important content of the GSW.

\section{WEB GEOINFIELLIGENCE}

From the Spatial Data Infrastructure (SDI) in the last century (Mohammadi et al., 2010), Spatial Data and Information Infrastructure (SDII) in the beginning of this century (Kiehle et al., 2007), CyberInfrastructure (Yang et al., 2010), to Geospatial Service Web (Gong et al., 2009), the transformation mechanism of Earth observation data - spatial information geographic knowledge has be consolidated in the network information infrastructure, and is involved into event sensing information extraction - knowledge services, Web GeoIntelligence has been the next step of GSW.

According to the current progress of geographic information scientific research, the Web-based geospatial intelligence can enhance and promote the level of geospatial information sharing and application service, by enabling them with automated reasoning, and building a powerful network of

\footnotetext{
* Corresponding author. Email: geopyue@gmail.com, Tel: +86-27-68778755
} 
intelligent geographical information analysis and decision support platform that incorporates latest progresses of networkbased multi-dimensional GeoInformation integration, and the development of Earth observation sensor Web to realize Webbased geospatial intelligence service (Figure 1).

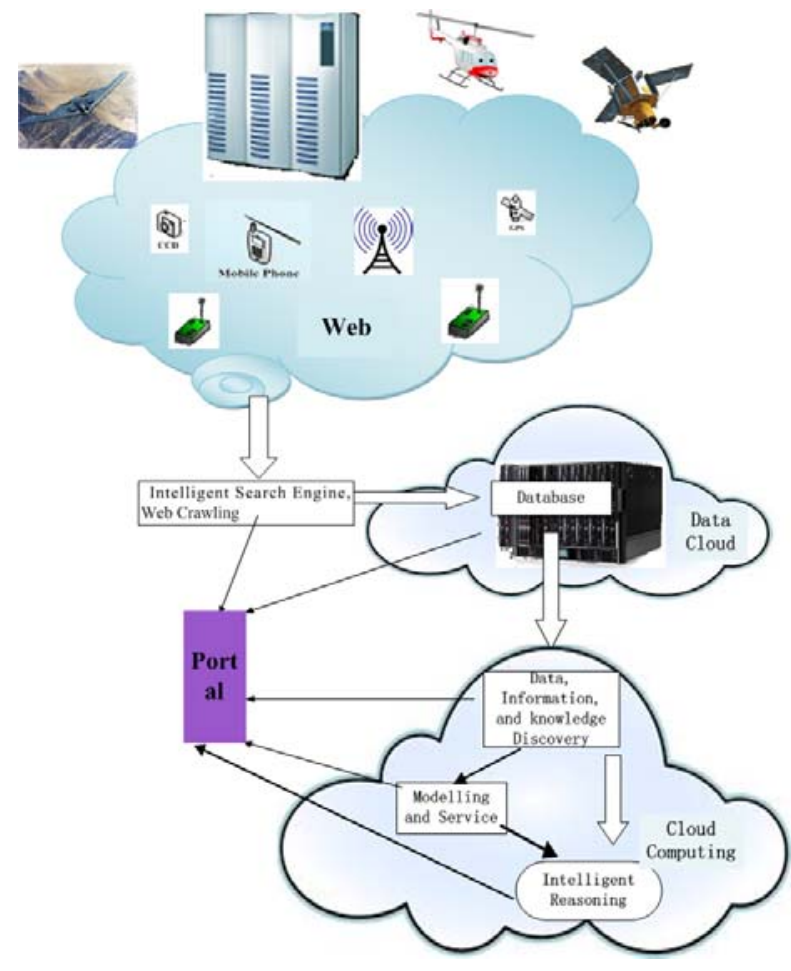

Figure 1. The concept of Web GeoIntelligence

The accomplishment of Web GeoIntelligence will build a new generation of multi-level, multi-granularity, multi-dimensional space-time data management and visualization platform, to realize the air, sky, earth and the sea, above and below ground, indoor and outdoor large-scale distributed data management and dynamic visualization; develop high precision and high efficiency of space-time analysis of algorithms and data modelling in the Web environment to create a Web analytics platform, based on the time autocorrelation (or space-time interactions) expression of multiple space-time-scale mathematical model of the physical equations and statistical analysis of the general principle; provide the intelligent Webbased services including the semantic integration of spatial information, solutions for intelligent modeling, automated geographic ontology mapping, spatial information semantic reasoning and knowledge mining, to realize integration and sharing of semantics of geospatial information in heterogeneous environments; establish a Web-based intelligent services platform, with multi-dimensional space-time visualization platform and the integration of Earth observation sensor networks to support geospatial real-time information service; provides multi-level, multi-granularity analysis and userassisted decision support capabilities.

\section{KEY ISSUES}

Several key issues need to be addressed to the development of GSW to fulfil Web GeoIntelligence. There issues are lists as follows.

\subsection{Web-based multi-dimensional space-time modelling and representation}

Based on the internet network, sensor network, network of things, mobile Internet and other Web networks, it is necessary to build a new generation of space-time data management and visualization platform with multi-level granularity and a full range of dimensions, and develop the distributed data management and dynamic visualization for data in space-earthsea, above and below ground, indoor and outdoor. For example, we can consolidate our development on the existing GeoGlobe platform and implement the network three-dimensional virtual globe systems for multi-source integration of heterogeneous information sharing and service. Some key issues in this direction include:

(1) representation of multidimensional spatial-temporal model in the Web environment

(2) Multi-dimensional space-time Web environment data modeling methods

(3) Storage and management of massive multi-source distributed three-dimensional world map data

(4) Network based three-dimensional rendering engine for Virtual Earth.

(5) Dynamic space-time data modeling and visualization

\subsection{Web-based multi-dimensional space-time data analysis}

Space-time data analysis models and methods need to be developed. In GSW, Web-based multi-dimensional space-time data analysis tools can help comprehensive utilization of geoscientific knowledge and Earth observation data. Key issues include:

(1) Representation of geoscientific processes and modeling of moving objects

(2) Numerical computation and optimization of geoscientific models

(3) Statistical analysis methodologies on space-time data

(4) Toolboxes for multi-dimensional space-time data analysis tools

\subsection{Web-based geospatial intelligent services}

The geospatial intelligent services can be provided in a Sensor Web environment. In the Sensor Web environment, sensor resource modeling and management, and real-time geospatial spatial information services need to be provided. The sensor observations can be used to support live geoprocessing and decision support. In geospatial resources query and decision support process, methodologies on searching, matching and reasoning need to be addressed. Through automatic/semiautomatic construction of process models to achieve efficient geospatial information search and distribution according to user needs, and use of spatial reasoning and knowledge mining in intelligent building of application models, a Web-based geospatial intelligence environment can be created. Key issues include:

(1) Resource description models and modeling approaches in the Earth observation sensor Web

(2) Real-time geospatial information services

(3) Intelligent discovery and matching of geospatial information

(4) Semantic integration, reasoning and knowledge mining of spatial information.

(5) Intelligent creation of geospatial information user models

(6) Evaluation and optimization of geospatial composite services 


\subsection{Integration platform}

The final goal will be a Web-based geospatial intelligence service platform, which can integrate multi-dimensional spacetime visualization and Earth observation sensor networks, provide real-time geospatial information services to support multi-level analyses in multi-granularity and decision support capabilities. Key issues include:

(1) Intelligent Geospatial Web Services Integration System

(2) Geographic information sharing, access, and interoperability based on virtualization technology

(3) Collaborative analysis of geoscientific data

\section{DEVELOPMENT APPROACHES}

Figure 2 shows the main components of development framework of the Web-based geospatial intelligence platform. The top level includes open collaborative research environment for end users and multi-dimensional spatial information integration environment. The middle layer is the world map public geographical information service platform. The middle layer includes components to support intelligent services such as spatial information service quality evaluation, spatial information processing tasks, spatial information integration, inference, and mining, and intelligent discovery and matching of spatial information. The bottom is a cloud computing management platform.

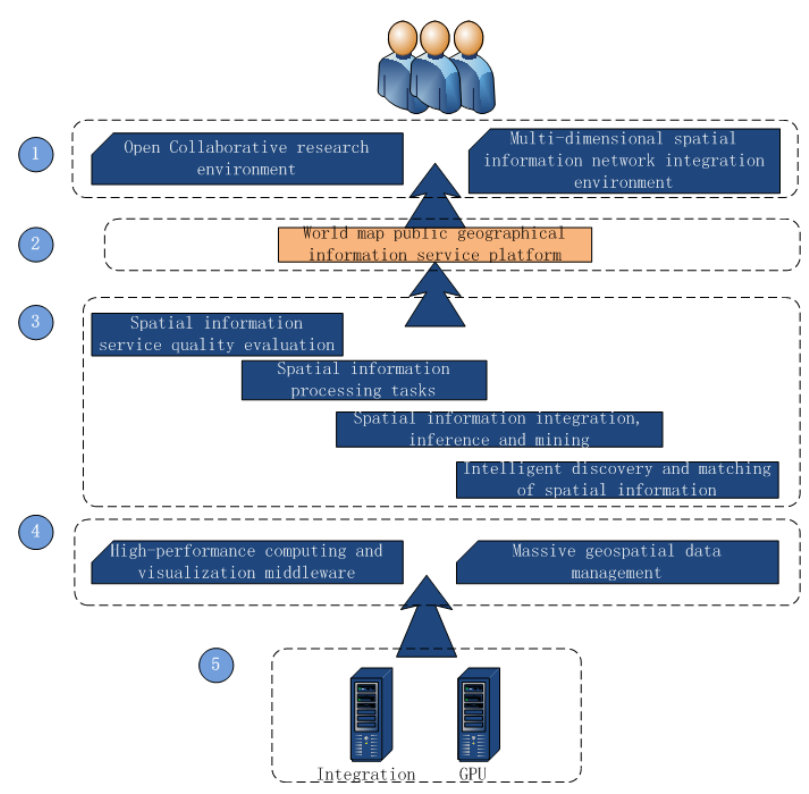

Figure 2 Web-based geospatial intelligence platform

The implementation will leverage the new and ongoing development in the Semantic Web, Artificial Intelligence (AI), Cloud and high performance computing technology, and Sensor Web etc. Semantic Web technologies (Berners-Lee et al., 2001), which give machine-processable meanings to the documents, allow the semantics of data and services machineunderstandable and thus can be used by machines (reasoning) for more effective discovery, automation, integration and reuse of geospatial data and services. The Semantic Web community works closely with AI community and there has been work in the Semantic Web community to apply ontology ideas developed in the AI community to various aspects of Web
Services and Web information search and manipulation. The emergence of Cloud Computing technology brings a new computing information technology (IT) infrastructure to general users. The key feature of the Cloud Computing technology is that it hides the underlying complexity of using IT resources, while at the same time bring the scalable, reliable, sustainable, ready-to-use, on-demand and cost-effective IT services to the general public (Armbrust et al., 2009). Thus, these technologies show considerable promise, and would allow representation and sharing of geospatial knowledge in a Web-based distributed environment.

The platform is built upon open, consensus-based standards for correctly transporting data, communicating information and evaluating results between the components of the framework. The standards cover international geographic information standards issued by ISO or OGC, Web service standards released by $\mathrm{W} 3 \mathrm{C}$ and other relative standards. Based on appropriate standards, the framework provides the "plug-inand-play" of community-developed, standard-compliant components and services.

\section{CONCLUSION}

This paper proposes the next step towards the establishment of Geospatial Service Web (GSW), namely the Web GeoIntelligence. Key issues and development approaches are addressed. By introducing intelligence into existing information infrastructure, the GSW will provide a powerful network platform consisting of intelligent geographical information analysis and decision support, and lower the threshold on the public use of geospatial information.

\section{ACKNOWLEDGEMENT}

This work is supported by National Basic Research Program of China (2011CB707105), Project 40801153 and 41023001 supported by NSFC, LIESMARS and SKLSE (Wuhan University) Special Research Funding. Discussions with colleagues in Spatial Information System Sector of LIESMARS are highly appreciated.

\section{REFERENCES}

Armbrust, M., Fox, A., Griffith, R., Joseph, Anthony D., Katz,Randy H., Konwinski, A., Lee, G., Patterson, D., Rabkin, A., Stoica, I., and Zaharia, M., 2009. Above the Clouds: A Berkeley View of Cloud Computing. Technical Report. EECS Department, University of California, Berkeley. 23 pp.

Berners-Lee, T., J. Hendler, and O. Lassila, 2001. The semantic web. Scientific American, 284(5), May 2001, pp. 34-43.

Gong, J., Wu, H., Gao, W., Yue, P., and Zhu, X., 2009. Geospatial Service Web. Geospatial Technology for Earth Observation, Li, D., Shan. J., and Gong, J. (Eds.), Springer Publication, USA. pp 355-379.

Kiehle, C., Heier, C., Greve, K., 2007. Requirements for next generation spatial data infrastructures-standardized web based geoprocessing and Web service orchestration. Transactions in GIS 11(6), 819-834.

Mohammadi, H., Rajabifard, A., Williamson, I.P., 2010. Development of an interoperable tool to facilitate spatial data 
International Archives of the Photogrammetry, Remote Sensing and Spatial Information Sciences, Volume XXXVIII-4/W25, 2011 ISPRS Guilin 2011 Workshop, 20-21 October 2011, Guilin, China

integration in the context of SDI. International Journal of Geographical Information Science 24(4), 487-505.
Yang, C., Raskin, R., Goodchild, M., Gahegan, M., 2010. Geospatial cyberinfrastructure: past, present and future. Computers, Environment and Urban Systems 34 (4), 264-277. 